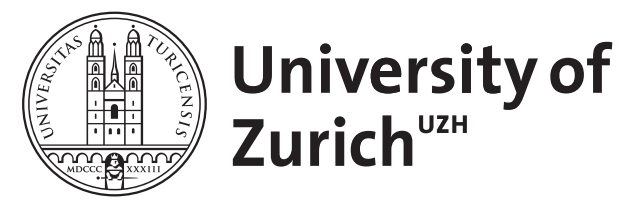

\title{
NMR spin-lattice relaxation rates in cuprates
}

\author{
Meier, P F
}

\begin{abstract}
An analysis of nuclear spin-lattice relaxation data in the normal state of cuprates that appropriately accounts for the highly anisotropic structures shows no contrasting temperature dependence of the $\mathrm{Cu}, \mathrm{O}$, and $\mathrm{Y}$ relaxations, which suggests that all nuclei relax by the same mechanism of the spin liquid. To investigate the temperature dependence of this mechanism, the model of fluctuating fields is used in which the rates are expressed in terms of hyperfine interaction energies and an effective correlation time eff characterizing the dynamics of the spin fluid. The former contain the effects of the antiferromagnetic static spin correlations, which cause the hyperfine field constants to be added more coherently at low temperature but incoherently at high temperature. At low temperatures, eff grows linearly with temperature as in ordinary metals. At high temperatures, however, the nuclear spin-lattice relaxation rates in various cuprates unequivocally reflect local moment features. This behavior is similar to that observed for the magnetic transition metals Fe, Co, and Ni, where also some properties show a cross-over from an itinerant behavior of delocalized electrons at low to that of localized moments at high temperatures
\end{abstract}

DOI: https://doi.org/10.1007/s10948-007-0285-9

Posted at the Zurich Open Repository and Archive, University of Zurich

ZORA URL: https://doi.org/10.5167/uzh-156396

Journal Article

Published Version

Originally published at:

Meier, P F (2008). NMR spin-lattice relaxation rates in cuprates. Journal of Superconductivity and Novel Magnetism, 21(1):17-20.

DOI: https://doi.org/10.1007/s10948-007-0285-9 


\title{
NMR Spin-Lattice Relaxation Rates in Cuprates
}

\author{
P.F. Meier
}

Received: 26 June 2007 / Accepted: 30 June 2007 / Published online: 19 September 2007

(C) Springer Science+Business Media, LLC 2007

\begin{abstract}
An analysis of nuclear spin-lattice relaxation data in the normal state of cuprates that appropriately accounts for the highly anisotropic structures shows no contrasting temperature dependence of the $\mathrm{Cu}, \mathrm{O}$, and $\mathrm{Y}$ relaxations, which suggests that all nuclei relax by the same mechanism of the spin liquid. To investigate the temperature dependence of this mechanism, the model of fluctuating fields is used in which the rates are expressed in terms of hyperfine interaction energies and an effective correlation time $\tau_{\text {eff }}$ characterizing the dynamics of the spin fluid. The former contain the effects of the antiferromagnetic static spin correlations, which cause the hyperfine field constants to be added more coherently at low temperature but incoherently at high temperature. At low temperatures, $\tau_{\text {eff }}$ grows linearly with temperature as in ordinary metals. At high temperatures, however, the nuclear spin-lattice relaxation rates in various cuprates unequivocally reflect local moment features. This behavior is similar to that observed for the magnetic transition metals $\mathrm{Fe}, \mathrm{Co}$, and $\mathrm{Ni}$, where also some properties show a cross-over from an itinerant behavior of delocalized electrons at low to that of localized moments at high temperatures.
\end{abstract}

Keywords Nuclear magnetic resonance - Cuprates . Spin-lattice relaxation - Antiferromagnetic correlations . Local moments

\section{Introduction}

Nuclear magnetic resonance (NMR) is a powerful probe for investigating the microscopic magnetic properties of

P.F. Meier $(\bowtie)$

Physics Institute, University of Zurich, 8057 Zurich, Switzerland e-mail: pfmeier@physik.uzh.ch cuprates that exhibit high-temperature superconductivity. An eminent advantage of this method is based on its highly local nature which allows one to get information about the distinct chemical species in the materials and make selective measurements of different crystallographic sites (for reviews, see Refs. [1, 2]).

The nuclear spin-lattice relaxation rate, ${ }^{k} T_{1 \alpha}^{-1}$ for a nuclear species $k$, is the rate at which the magnetization relaxes to its equilibrium value in the external magnetic field applied in direction $\alpha$. The relaxation of the nuclei under consideration in the cuprates is caused by two or more fluctuating hyperfine fields that originate from magnetic moments localized near the coppers. Since the squares of these fields come into play, one of the first tasks when interpreting spin-lattice relaxation data is to determine whether the hyperfine fields should be added coherently or incoherently at the nucleus. While Mila and Rice [3] added them incoherently, Monien, Pines and Slichter [4] considered both extreme cases, and from the analysis of the copper data in $\mathrm{YBa}_{2} \mathrm{Cu}_{3} \mathrm{O}_{7}$, concluded that within a one-component model, the fields should be added coherently. The question of coherency was put aside when Millis, Monien and Pines (MMP) gave a quantitative and complete phenomenological description of the relevant measurements by putting forward a model $[5,6]$ where the nuclear spin-lattice relaxation rate, via the fluctuation-dissipation theorem, is expressed in terms of the low-frequency limit of the imaginary part of the spin susceptibility $\chi$ which is split into two parts, $\chi=\chi_{\mathrm{AF}}+\chi_{\mathrm{FL}}$. The first term, $\chi_{\mathrm{AF}}$ represents the anomalous contribution to the spin system and is peaked at or near $\vec{Q}=(\pi / a, \pi / a)$. The second term, $\chi_{\mathrm{FL}}$, is a parameterized form of the normal Fermi liquid contribution. For copper, the contributions from the Fermi liquid part are much smaller than those from $\chi_{\mathrm{AF}}$ but they dominate the relaxation of oxygen and yttrium nuclei. In the parameterization for $\chi$ introduced by MMP, 
$\chi_{\mathrm{AF}}$ is strongly peaked at $\vec{Q}$ and the hyperfine fields at the copper are added essentially coherently. On the other hand, for any $\vec{q}$-independent $\chi_{\mathrm{FL}}$, the contributions of these fields are strictly incoherent, and the fields at the oxygen, are therefore added incoherently in the MMP theory.

This coherency is related to the short range antiferromagnetic (AFM) correlations. To investigate its temperature dependence in detail, we have reanalyzed [7] various NMR relaxation rate data with the goal to get the information on the degree of coherency directly from the data. The premises of our different approach to the analysis of spin-lattice relaxation data in the normal state of cuprates are the following. We go back to the simple model of fluctuating fields [8]. We retain, however, the AFM correlations which are an essential feature of the concept of the nearly AFM Fermi liquid. The normalized AFM spin-spin correlations between adjacent coppers are a key quantity, since they determine to what extent the hyperfine fields are added coherently.

Since cuprates are very anisotropic materials, it is instructive to describe the spin-lattice relaxation rates ${ }^{k} T_{1 \alpha}^{-1}$ for the nuclear species $k$ with $\alpha$ denoting the direction of the applied static field in the following way

${ }^{k} T_{1 \alpha}^{-1}={ }^{k} U_{\beta}+{ }^{k} U_{\gamma}$

where $\beta$ and $\gamma$ are the two orthogonal directions perpendicular to $\alpha$. The quantity ${ }^{k} U_{\beta}$ describes then the contribution to ${ }^{k} T_{1 \alpha}^{-1}$ and ${ }^{k} T_{1 \gamma}^{-1}$ caused by fluctuating fields along the crystallographic direction $\beta$. These transformed rates ${ }^{k} U_{\beta}$, which in the following will just be called rates, are not directly accessible by experiment except for particular symmetries (e.g., ${ }^{63} U_{a b}={ }^{63} T_{1 c}^{-1} / 2$ ), but in general can be obtained if a complete set of data measured with the applied field along all three crystallographic axes is available.

For cuprates, the most complete set of NMR data with respect to different nuclei and directions of external field relative to the crystallographic axes is available for optimally doped $\mathrm{YBa}_{2} \mathrm{Cu}_{3} \mathrm{O}_{7}$ in the normal state between $100 \mathrm{~K}$ and room temperature. One particular aspect that absorbed the attention of the NMR community is that these experiments show a dramatic contrast in the temperature dependence of ${ }^{k} T_{1 \alpha}^{-1}$ between copper and oxygen sites in the $\mathrm{CuO}_{2}$-plane although these sites lie less than $2 \AA$ apart. However, the temperature dependence of the transformed rates ${ }^{k} U_{\alpha}$ exhibits no drastic contrast between $\mathrm{Cu}, \mathrm{O}$, and $\mathrm{Y}$. They grow with increasing $T$, as is expected for fluctuations, and the values for ${ }^{63} U_{\alpha}$ are of the same order of magnitude as for metallic copper. This suggests, therefore, that all nuclei under consideration relax in a similar fashion by the same mechanism of the spin liquid.

To investigate this mechanism, the model of fluctuating fields [8] is used in which the NMR spin-lattice relaxation rate is expressed as

${ }^{k} T_{1 \alpha}^{-1}(T)=\left[{ }^{k} V_{\beta}(T)+{ }^{k} V_{\gamma}(T)\right] \tau_{\mathrm{eff}}(T)$.
The term $\tau_{\text {eff }}(T)$ is an effective electronic spin-spin correlation time, and ${ }^{k} V_{\beta}(T)$ and ${ }^{k} V_{\gamma}(T)$ correspond to the square of the components $\beta$ and $\gamma$ of the hyperfine fields at the nucleus. For a planar oxygen, the fluctuating fields stem from the localized moments on the two copper moments (at site 0 and 1 ) adjacent to the $\mathrm{O}$ and one gets

${ }^{17} V_{\beta}(T)=\frac{1}{4 \hbar^{2}} 2 C_{\beta}^{2}\left[1+K_{01}^{\beta}(T)\right]$

where $C_{\beta}$ is the hyperfine parameter and $K_{01}^{\beta}$ is the $\beta$ component of the normalized "static" spin-spin correlation defined as

$K_{01}^{\beta}(T)=4\left\langle S_{0}^{\beta} S_{1}^{\beta}\right\rangle$

and can take values between -1 (fully antiferromagnetically correlated and yielding ${ }^{17} V_{\beta}=0$ ) and 0 (no correlation).

For the planar coppers, there are, besides the on-site hyperfine field $A_{\beta}$, isotropic hyperfine fields $B$ transferred from the four nearest neighbor copper ions, and therefore higher order AFM correlations $K_{i j}^{\beta}$ are of importance. For simplicity, it is assumed that these correlations decay exponentially with distance and can therefore be expressed in terms of $K_{01}^{\beta}$, i.e. $K_{12}^{\beta}=\left|K_{01}^{\beta}\right|^{\sqrt{2}}$ and $K_{13}^{\beta}=\left|K_{01}^{\beta}\right|^{2}$.

Equations (2-4) emphasize the different temperature dependencies that determine ${ }^{k} T_{\alpha}^{-1}(T)$. The ${ }^{k} V_{\beta}(T)$, which apart from the factor $\hbar^{2}$ are the static hyperfine energies squared, vary with temperature due to changes of the static spin correlations $K_{01}^{\beta}(T)$, whereas the effective correlation time $\tau_{\text {eff }}(T)$ reflects the changes in the dynamics of the spin fluid. To extract the important physics of the spin liquid from the NMR data, it is essential to disentangle these two different temperature dependencies.

The determination of the temperature dependence of $K_{01}^{\beta}(T)$ from the data for $\mathrm{YBa}_{2} \mathrm{Cu}_{3} \mathrm{O}_{7}$ gives [7] -0.5 at $T=100 \mathrm{~K}$ with only a slight anisotropy between the inand out-of-plane components. This corresponds to a correlation length of about one lattice constant. With increasing temperatures, the AFM correlations become weaker and one gets -0.1 at $T=600 \mathrm{~K}$.

Once the temperature dependence of $K_{01}^{\beta}(T)$ is determined and the hyperfine parameters known, $(2-4)$ can be applied to each of the seven sets of data for ${ }^{k} U_{\alpha}(T)\left[{ }^{63} U_{c}(T)\right.$, ${ }^{63} U_{a b}(T),{ }^{89} U_{c}(T),{ }^{89} U_{a b}(T)$, and 3 for the planar O] to obtain $\tau_{\text {eff. }}$. These seven curves for $\tau_{\text {eff }}$ nearly coincide and the temperature dependence of the average $\tau_{\text {eff }}$ is well fit by a function of the form

$\tau_{\text {eff }}^{-1}=\tau_{1}^{-1}+\tau_{2}^{-1}$

with

$\tau_{1}=a T \quad$ and $\quad \tau_{2}=$ const. 


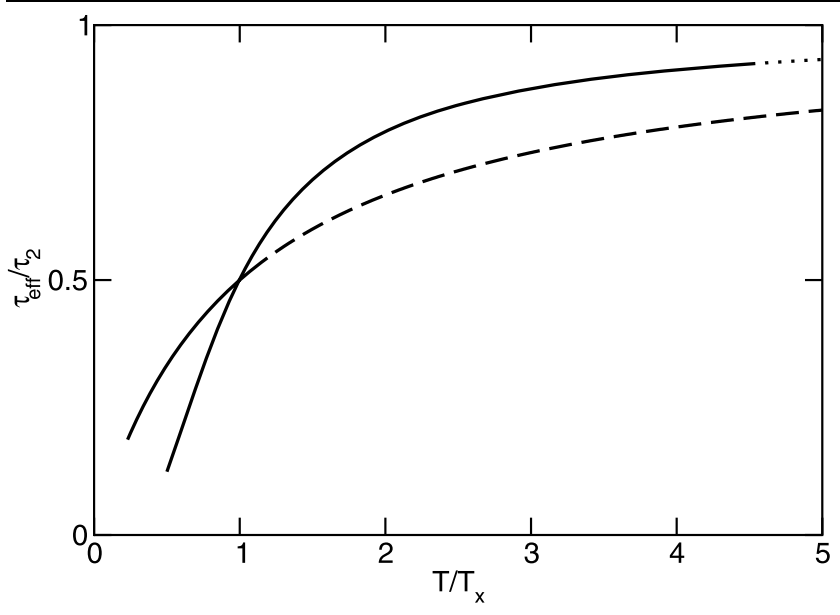

Fig. 1 Normalized relaxation rates $\tau_{\text {eff }} / \tau_{2}$ versus $T / T_{x}$ from measurements in $\mathrm{YBa}_{2} \mathrm{Cu}_{3} \mathrm{O}_{7}$ (solid line from 0.23 to 1.19) and $\mathrm{YBa}_{2} \mathrm{Cu}_{4} \mathrm{O}_{8}$ (solid line from 0.50 to 4.54 ) and extrapolations (dashed and dotted) to higher temperatures

We obtain $a=7.0 \times 10^{-18} \mathrm{~s} / \mathrm{K}$ and the temperature independent $\tau_{2}=3.0 \times 10^{-15} \mathrm{~s}$. These values allow us to compare the model predictions with selected data obtained at higher temperatures (up to $500 \mathrm{~K}$ for ${ }^{63} T_{1 c}^{-1}$ and up to $700 \mathrm{~K}$ for ${ }^{17} T_{1 c}^{-1}$ and ${ }^{89} T_{1 c}^{-1}$ ). The agreement is excellent for $\mathrm{Cu}$ and good for $\mathrm{O}$ and $\mathrm{Y}$.

For low temperatures, the spin-lattice relaxation rates in $\mathrm{YBa}_{2} \mathrm{Cu}_{3} \mathrm{O}_{7}$ are thus dominated by $\tau_{1}=a T$ as in simple metals. If a degenerate Fermi gas is employed, the value of $a$ corresponds to a Fermi temperature of $1050 \mathrm{~K}$. At elevated temperatures, a deviation from this linear $T$-dependence occurs. Defining a cross-over temperature $T_{x}$ by $\tau_{1}\left(T_{x}\right)=\tau_{2}$ one finds $T_{x}=420 \mathrm{~K}$. This cross-over will be further discussed below. In Fig. 1, the normalized temperature dependence of $\tau_{\text {eff }} / \tau_{2}$ is shown versus $T / T_{x}$. The solid line (from 0.23 to 1.19 ) is the result of the analysis of the data from $T=96 \mathrm{~K}$ to $500 \mathrm{~K}$. The dashed line is the extrapolation to higher temperatures according to (5a).

For underdoped compounds, less complete NMR data sets are available. A fit of the spin-lattice relaxation experiments in $\mathrm{YBa}_{2} \mathrm{Cu}_{3} \mathrm{O}_{6.63}$ gives somewhat stronger AFM correlation, as expected, with $K_{01}^{\beta}(T) \approx-0.65$ at $T=100 \mathrm{~K}$. The extracted correlation times could not be fitted with the function (5). However, the same ansatz

$\tau_{\mathrm{eff}}^{-1}=\tau_{1}^{-1}+\tau_{2}^{-1}$

but with

$\tau_{1}=a T e^{-g / T} \quad$ and $\quad \tau_{2}=\mathrm{const}$

provides a very good fit with the values $a=10 \times 10^{-18} \mathrm{~s} / \mathrm{K}$, $g=97 \mathrm{~K}, \tau_{2}=2.9 \times 10^{-15} \mathrm{~s}$, and a cross-over temperature $T_{x}=375 \mathrm{~K}$. The gap $g$ introduced here is of course connected with the spin gap in the excitation spectrum which in the NMR literature, however, is defined in quite a different way.

In stoichiometric $\mathrm{YBa}_{2} \mathrm{Cu}_{4} \mathrm{O}_{8}$, NMR lines are much narrower than in other cuprates and allow precise measurements. In particular, the rates ${ }^{63} T_{1 c}^{-1}$ have been determined $[9,10]$ up to $700 \mathrm{~K}$ and can be excellently fitted with a correlation time given by (6) with $g=195 \mathrm{~K}$, $\tau_{2}=1.9 \times 10^{-15} \mathrm{~s}$ and $T_{x}=154 \mathrm{~K}$. The solid line in Fig. 1 (from 0.50 to 4.54 ) is the result of the analysis of the $\mathrm{Cu}$ data between $77 \mathrm{~K}$ and $700 \mathrm{~K}$.

A large amount of NMR data exist also for $\mathrm{La}_{2-x} \mathrm{Sr}_{x}-$ $\mathrm{CuO}_{4}$ for various doping levels $x$. Above $100 \mathrm{~K}$, the relaxation rates ${ }^{63} T_{1 c}^{-1}$ become nearly independent of temperature. Our model is in agreement with this behavior if the cross-over temperature $T_{x}<100 \mathrm{~K}$. Indeed, a fit of (6) to the data for $\mathrm{La}_{1.85} \mathrm{Sr}_{0.15} \mathrm{CuO}_{4}$ gives $T_{x}=70 \mathrm{~K}$ and $\tau_{2}=2.9 \times 10^{-15}$ s. Imai et al. [11] found that at high temperatures $(\sim 800 \mathrm{~K})$ the rates of the doped metallic samples were nearly identical to that of the undoped sample, which is an insulator with a magnetic behavior that can well be described by a two-dimensional Heisenberg antiferromagnet. For the latter, Moriya [12] had calculated $T_{1}^{-1}$ for a nucleus of a magnetic ion treating the exchange interaction within the model of a Gaussian random process with characteristic frequency $\omega_{e}$.

The same identical behavior of the insulating and metallic materials at high temperatures is also found in the $\mathrm{YBaCuO}$ family. For the insulating parent compound $\mathrm{YBa}_{2} \mathrm{Cu}_{3} \mathrm{O}_{6}$, Pozzi et al. [13] reported that in the paramagnetic phase ${ }^{63} T_{1 c}^{-1}$ declines with increasing temperature and levels off between 800 and $900 \mathrm{~K}$ at a value of $\sim 2000 \mathrm{~s}^{-1}$. In the metallic underdoped compound $\mathrm{YBa}_{2} \mathrm{Cu}_{4} \mathrm{O}_{8}$, Zimmermann et al. [9] measured a value of about $1800 \mathrm{~s}^{-1}$ at $750 \mathrm{~K}$. The same value is also obtained [14] for optimally doped $\mathrm{YBa}_{2} \mathrm{Cu}_{3} \mathrm{O}_{7}$ at the highest measured temperature of $500 \mathrm{~K}$.

Within our model, the copper NMR relaxation rate simplifies for high temperatures to

${ }^{63} T_{1 c}^{-1}(T \rightarrow \infty)=\frac{2}{4 \hbar^{2}}\left(A_{a b}^{2}+4 B^{2}\right) \tau_{2}$

since the hyperfine fields are added incoherently and $\tau_{\text {eff }} \approx$ $\tau_{2}$. The values for the hyperfine interaction energy parameters are only approximately known. It is generally agreed, however, that $\left|A_{a b}\right|$ is much smaller than $B$ such that ${ }^{63} T_{1 c}^{-1}(T \rightarrow \infty) \approx 2(B / \hbar)^{2} \tau_{2}$. With $B=0.47 \mu \mathrm{eV}$ (Ref. [15]) and $\tau_{2}=2.9 \times 10^{-15}$ one gets ${ }^{63} T_{1 c}^{-1}(T \rightarrow$ $\infty)=2900 \mathrm{~s}^{-1}$ for $\mathrm{La}_{2} \mathrm{CuO}_{4}$ and a smaller value for $\mathrm{YBa}_{2} \mathrm{Cu}_{3} \mathrm{O}_{6}$ with a reduced value for the transferred hyperfine field $B=0.40 \mu \mathrm{eV}$.

In conclusion, a large amount of NMR spin-lattice relaxation rates in cuprates can be explained by a robust model 
of fluctuating hyperfine fields. When the anisotropic structures are properly accounted for, there remains no striking difference between the relaxations observed for the different nuclei. The hyperfine fields at a nucleus are added incoherently at high temperatures. The degree of coherency at lower $T$ is determined by the AFM spin correlations which at least for $\mathrm{YBa}_{2} \mathrm{Cu}_{3} \mathrm{O}_{7}$ can be extracted from the data. The remaining basic relaxation mechanism which exchanges energy between the nuclear spins and the quasi-localized moments generally has a temperature dependence given by $\tau_{\text {eff }}^{-1}=\tau_{1}^{-1}(T)+\tau_{2}^{-1}$. At low $T$, the term $\tau_{1}$ dominates which is linear in $T$ (modified sometimes with a sort of gap). A cross-over then occurs at $T_{x}$ to a constant $\tau_{2}$ at high $T$. The cross-over temperature $T_{x}$ is below $100 \mathrm{~K}$ for $\mathrm{La}_{2} \mathrm{CuO}_{4}$, $154 \mathrm{~K}$ for $\mathrm{YBa}_{2} \mathrm{Cu}_{4} \mathrm{O}_{8}$, and $420 \mathrm{~K}$ for $\mathrm{YBa}_{2} \mathrm{Cu}_{3} \mathrm{O}_{7}$. In the case of $\mathrm{La}_{1.85} \mathrm{Sr}_{0.15} \mathrm{CuO}_{4}$, the constant $\tau_{2}$ is thus dominating the relaxation already at temperatures above $100 \mathrm{~K}$ and has a value which coincides with that of the rate with which a copper nucleus flips in the exchange field of the paramagnetic regime of the AFM insulator $\mathrm{La}_{2} \mathrm{CuO}_{4}$. The same identical behavior of the insulating paramagnetic and the metallic phase occurs also in the $\mathrm{YBaCuO}$ family.

It has to be emphasized that this functional form of $\tau_{\text {eff }}$ is not that expected for a two-fluid model. It is rather a onecomponent fluid which at low temperatures reveals a metallic and itinerant character of charge carriers but exhibits with increasing $T$ more local features. In other words, the doped holes dominate the NMR spin-lattice relaxation rates at low temperatures by energy exchange with the nucleus by a mechanism with effective correlation time $\tau_{1}$ which increases with temperature. When it becomes larger than the constant $\tau_{2}$, the nuclei relax due to the hyperfine interactions produced by the local moments in the same way as the nuclei in the insulating paramagnetic phases of the parent substances do.

This cross-over from an itinerant behavior at lower temperatures to the appearance of localized moments at elevated $T$ is similar [16] to that observed for some properties of the ferromagnetic transition metals $\mathrm{Fe}, \mathrm{Co}$, and $\mathrm{Ni}$. With increasing temperatures, transition metals show more and more local features, while itineracy and delocalization of the d electrons become less important. The magnetization curves closely resemble Brillouin curves and the susceptibility in the paramagnetic regime does not show a Pauli-like but a Curie-Weiss-like behavior.

The measured NMR spin-lattice relaxation rates thus provide rich information about the temperature dependence of the electronic and spin excitations which, however, still awaits a complete understanding. In this respect, it should also be mentioned that the analysis [17] of ${ }^{k} T_{1 \alpha}^{-1}$ data in the superconducting state within the frame of our model reveals surprising results about the symmetry of spin correlations.

Acknowledgements This work has been performed in collaboration with Anne-Christine Uldry. We are grateful to M. Mali, J. Roos, D. Brinkmann, and C.P. Slichter for inspiring discussions.

\section{References}

1. Brinkmann, D., Mali, M.: NMR Basic Principles and Progress, vol. 31. Springer, Heidelberg (1994)

2. Rigamonti, A., Borsa, F., Caretta, P.: Rep. Prog. Phys. 61, 1367 (1998)

3. Mila, F., Rice, T.M.: Physica C 157, 561 (1989)

4. Monien, H., Pines, D., Slichter, C.P.: Phys. Rev. B 41, 11120 (1990)

5. Millis, A.J., Monien, H., Pines, D.: Phys. Rev. B 54, 167 (1990)

6. Zha, Y., Barzykin, V., Pines, D.: Phys. Rev. B 54, 7561 (1996)

7. Uldry, A., Meier, P.F.: Phys. Rev. B 72, 094508 (2005)

8. Slichter, C.P.: Principles of Magnetic Resonance. Springer, Berlin (1996)

9. Zimmermann, H., Mali, M., Brinkmann, D., Karpinski, J., Kaldis, E., Rusiecki, S.: Physica C 159, 681 (1989)

10. Curro, N.J., Imai, T., Slichter, C.P., Dabrowski, B.: Phys. Rev. B 56, 877 (1997)

11. Imai, T., Slichter, C.P., Yoshimura, K., Kosuge, K.: Phys. Rev. Lett. 70, 1002 (1993)

12. Moriya, T.: Prog. Theor. Phys. 16, 641 (1993)

13. Pozzi, R., Mali, M., Brinkmann, D., Erb, A.: Phys. Rev. B 60, 9650 (1999)

14. Barrett, S.E., et al.: Phys. Rev. Lett. 66, 108 (1991)

15. Haase, J., Slichter, C.P., Stern, R., Millings, C.T., Hinks, D.G.: J. Supercond. Incorp. Novel Magn. 13, 723 (2000)

16. Fulde, P.: Electron Correlations in Molecules and Solids. Springer Series in Solid-State Sciences, vol. 100. Springer, Berlin (1995)

17. Uldry, A., Mali, M., Roos, J., Meier, P.F.: J. Phys. Condens. Matter 17, L499 (2005) 